\title{
Quarkonia Production at Fermilab
}

\author{
A. Sansoni \\ For the CDF Collaboration \\ Fermi National Accelerator Laboratory \\ P.O. Box 500, Batavia, Illinois 60510 \\ Istitute Nazionale di Fisica Nucleare \\ Frascati (RM) Italy
}

\begin{abstract}
August 1995
Published Proceedings from the 6th International Symposium on Heavy Flavour Physics, Pisa, Italy, June 6-11, 1995.
\end{abstract}




\section{Disclaimer}

This report was prepared as an account of work sponsored by an agency of the United States Government. Neither the United States Government nor any agency thereof, nor any of their employees, makes any warranty, express or implied, or assumes any legal liability or responsibility for the accuracy, completeness, or usefulness of any information, apparatus, product, or process disclosed, or represents that its use would not infringe privately owned rights. Reference herein to any specific commercial product, process, or service by trade name, trademark, manufacturer, or otherwise, does not necessarily constitute or imply its endorsement, recommendation, or favoring by the United States Government or any agency thereof. The views and opinions of authors expressed herein do not necessarily state or reflect those of the United States Government or any agency thereof. 
CDF/PHYS/BOTTOM/PUBLIC/3270

Proceedings: HF95 Pisa.

August 14, 1995

\title{
Quarkonia production at Fermilab
}

\author{
CDF Collaboration
}

A.Sansoni

Istituto Nazionale di Fisica Nucleare, Frascati (RM) Italy.

\begin{abstract}
Recent data on $J / \psi, \psi(2 S), \chi_{c}$ and $\Upsilon$ production has unraveled unexpected features of $Q \bar{Q}$ bound states production in high energy hadronic collisions.

In the 1992-1995 runs CDF has collected large samples of $J / \psi, \psi(2 S)$ and $\Upsilon$ identified through their muonic decay. In the charmonium system all production sources have been separately measured and compared with the theoretical predictions. A large excess of direct production has been observed for both $\psi(2 S)$ and $J / \psi$. The relative production rate for the $\chi_{c}^{1}$ and $\chi_{c}^{2}$ has also been measured.

Results on $J / \psi, \psi(2 S)$ and $\chi_{c}$ production from the fixed target experiments E789,E705 and E672/706 are also presented. Important features of the production of charmonium states are shared by fixed target and collider data.
\end{abstract}

\section{Introduction}

The production of $Q \bar{Q}$ bound states in high energy collisions has recently been the subject of renewed theoretical interest. This is due to the fact that the experiments have measured the production cross sections for a wide variety of $Q \bar{Q}$ states and, for the $c \bar{c}$ system, they have been able to disentangle all the sources of production thus allowing a strict comparison with the theoretical prediction.

The large mass of the $c$ quark $\left(m_{c} \sim 1.5 \mathrm{GeV} / c^{2}\right)$ implies that bound $c \bar{c}$ states are the first systems where perturbative $Q C D$ is expected to be applicable. At leading order, $O\left(\alpha_{s}^{3}\right)$, the production of a quarkonium state is described by the production of a free, color singlet, $Q \bar{Q}$ pair with the same quantum numbers of the quarkonium state [1]. The formation of the bound state is a non perturbative process that can be factored into a parameter either calculable within potential models or extracted from experimental data. Recently this picture has been improved with the inclusion of the 
process where a high $p_{T}$ parton fragments into a color singlet $Q \bar{Q}$ pair [2]. This is an $O\left(\alpha_{s}^{4}\right)$ process but the extra $p_{T}^{2} / m_{Q}^{2}$ factor makes this the dominant one at high $p_{T}$. In the $c \bar{c}$ system, high energy collider and fixed target experiments have measured the production of $J / \psi, \psi(2 S)$ and $\chi_{c}$ states. The predictions based on this model $[1,3]$ were that the $\chi_{c}$ have by far the largest cross section. Direct production of both $J / \psi$ and $\psi(2 S)$ was expected to be small because the extra gluon directly coupled to the $c \bar{c}$ line suppress this diagram. The $\chi_{c}^{0}$ has a negligible branching ratio into $J / \psi$ therefore prompt $J / \psi$ were expected to originate almost completely from the radiative decays of the $\chi_{c}^{1}$ and $\chi_{c}^{2}$. Feed-down is not relevant for the $\psi(2 S)$ since the $\psi(2 S)$ is heavier than the $\chi_{c}$ 's.

In collider experiments $c \bar{c}$ states can also arise from the decay of $b$ flavored hadrons (this mechanism is negligible for fixed target experiments). This, non-prompt component, was expected to be the dominant source of $\psi(2 S)$ and to contribute significantly to $J / \psi$ production.

Prior to the successful operation of the CDF Silicon Vertex Detector, collider data were not inconsistent with this picture [4]. Fixed target and ISR collider experiments (no $b$ production), on the other hand, reported already many years ago that $\chi_{c}$ 's were not the primary source of $J / \psi[5]$ in disagreement with the expectations of the color singlet QCD model.

\section{Quarkonia production at CDF}

The data presented here are from $p \bar{p}$ collisions at $\sqrt{s}=1.8 \mathrm{TeV}$ collected with the CDF detector in the 1992-1995 runs. The detector has been described in detail elsewhere [6]. The features most important for these analysis are: 1) the large tracking system contained in the $1.4 T$ axial magnetic field providing the high resolution momentum measurement of charged particles, 2) the 4-layer Silicon Vertex Detector (SVX) [7] providing the identification of secondary vertices associated with $b$ decays, 3 ) the $\mathrm{Pb}$-scintillator central electromagnetic calorimeter with a strip chamber embedded at a depth of 5.9 radiation lengths used for the photon reconstruction and 4) the muon chamber system, surrounding the central calorimeter, for the muon identification in the range $|\eta|<1.0$.

The $J / \psi, \psi(2 S)$ and $\Upsilon$ 's are reconstructed using their muonic decay. The cutoff associated with the steel of the central calorimeter is $p_{T}(\mu)>1.5 \mathrm{GeV} / \mathrm{c}$. The level 1 trigger efficiency rises from $50 \%$ at $p_{T}(\mu)=1.6 \mathrm{GeV} / \mathrm{c}$ to $90 \%$ at $p_{T}(\mu)=$ $3.1 \mathrm{GeV} / \mathrm{c}$. This implies that only relatively high $p_{T} J / \psi$ can be reconstructed while, due to its larger mass, $\Upsilon$ 's can be reconstructed down to almost zero $p_{T}$. The event selection required:

- Two opposite sign good quality central muons

- $p_{T}>2.0 \mathrm{GeV} / \mathrm{c}$ for the soft $\mu$ 
- $p_{T}>2.8 \mathrm{GeV} / \mathrm{c}$ for the hard $\mu$

- $p_{T}^{\mu \mu}>4.0 \mathrm{GeV} / c$ and $|\eta|<0.6$ for $J / \psi$ and $\psi(2 S)$

- $p_{T}^{\mu \mu}>0.5 \mathrm{GeV} / \mathrm{c}$ and $|y|<0.4$ for $\Upsilon$ 's

After this selection we observe a very clean signal of about $180,000 \mathrm{~J} / \psi$ in $75 \mathrm{pb}^{-1}$ of data. Cross sections are obtained correcting for acceptance and reconstruction efficiencies. The geometric and kinematical acceptances are determined from Monte Carlo simulations. The trigger and reconstruction efficiencies are measured from the data.

\section{$2.1 \psi(2 S)$}

We first review the $\psi(2 S)$. In $18 \mathrm{pb}^{-1}$ we observe $896 \pm 94 \psi(2 S) \rightarrow \mu^{+} \mu^{-}$， with a signal to background ratio of 0.57 . The measured production cross section is $\sigma\left(|\eta|<0.6, p_{t}>4.0 \mathrm{GeV} / \mathrm{c}\right)=(94 \pm 8 \pm 9.3 \pm 21(B r)) \mathrm{nb}$ where $(B r)$ is the systematic uncertainty associated with the decay branching ratio.

The first problem consists in separating between prompt production and feeddown from $b$ decays. In the previous analysis [4] it was impossible to do this experimentally, therefore, following the theoretical prejudice, it was assumed that all $\psi(2 S)$ came from $b$ decays and $b$ a cross section was derived. Now, with the CDF Silicon Vertex Detector, the prompt and long lived component can be precisely measured.

For each $\psi(2 S)$ candidate reconstructed in the SVX the two dimensional decay length $L_{x y}$ is calculated. $L_{x y}$ is the projection of the vector $\vec{X}$, pointing from the primary to the secondary vertex, onto the transverse momentum vector of the $\psi(2 S)$. The position of the secondary vertex is obtained by constraining the two muons to come from a common decay point. To convert $L_{x y}$ into a proper lifetime we use $(\beta \gamma)$ of the $\psi(2 S)$ and a correction factor $F_{\text {corr }}$, determined from Monte Carlo, to take into account the difference with $(\beta \gamma)$ of the $b$ hadron.

$$
\begin{gathered}
L_{x y}=\vec{X} \cdot \vec{P}_{t} / P_{t} \\
c \tau_{p s e u d o}=\frac{L_{x y}}{\left(P_{t} / M\right) \cdot F_{c o r r}}
\end{gathered}
$$

Figure 1 shows the $c \tau_{\text {pseudo }}$ distribution for the $\psi(2 S)$. To determine the fraction from $b$ 's we fit this distribution with the sum of three contributions: 1) the prompt component parametrized with a gaussian, 2) the long lived component parametrized with an exponential smeared with the detector resolution (hatched in Fig.1) and 3) 
the background component obtained from the sidebands of the $\psi(2 S)$ (dark shading in Fig.1). The result is that $22.8 \pm 3.5 \%$ of the $\psi(2 S)$ come from $b$ decays, by far the majority is unambiguously prompt.

The statistics are sufficient to do this fit in several $p_{T}$ bins, the fraction of $\psi(2 S)$ from $b$ 's slowly increase with $p_{T}$. From this measurement we derive the differential cross section for $\psi(2 S)$ from $b$ 's and for prompt $\psi(2 S)$. This is shown in Figure 2 together with the theory curves. The $b$ component is in reasonable agreement with the NLO QCD theory prediction while the prompt component is about a factor of 50 over the theoretical curve [3].

This large discrepancy, nicknamed the "CDF $\psi(2 S)$ anomaly", has prompted the proposal that charmonium states above the $D \bar{D}$ threshold, whose decay in open charm is forbidden, or new exotic states, decay in $\psi(2 S) X$ accounting for the excess [8]. Another proposal is that an additional mechanism, such as the production of a color octet $c \bar{c}$ state evolving non-perturbatively into a $\psi(2 S)$ bound state, must be present in charmonium production [9]. To better understand the origin of this discrepancy it is natural, from the experimental point of view, to see if this excess is a feature of the $\psi(2 S)$ only or it is also found in $J / \psi$ production.

\section{$2.2 J / \psi$}

For the $J / \psi$ the integrated production cross section is $\sigma\left(|\eta|<0.6, p_{t}>4.0 \mathrm{GeV} / \mathrm{c}\right)=$ $\left(487 \pm 3_{-47}^{+51} \pm 20(B r)\right) \mathrm{nb}$. The prompt and $b$ component are measured with the SVX fitting the $c \tau_{\text {pseudo }}$ distribution in the same way described for the $\psi(2 S)$. The fraction of $J / \psi$ from $b$ 's is $19.6 \pm 1.5 \%$, similar to the $\psi(2 S)$, and the cross section of $J / \psi$ from $b$ 's is also in decent agreement with theory. Combining the cross section and fraction measurements for $J / \psi$ and $\psi(2 S)$ we derive the ratio of cross sections of $\sigma\left(\psi^{\prime}\right) / \sigma(\psi)=0.19 \pm 0.05$ for promptly produced $\psi(2 S)$ and $J / \psi$ with $p_{t}>4.0 \mathrm{GeV} / \mathrm{c}$ and $|\eta|<0.6$.

The prompt $J / \psi$ cross section is again underestimated by the theoretical prediction, although only by a factor of $\sim 6$. But in this case the the prompt component includes both direct $J / \psi$ production and feed-down from $\chi_{c}$. It is therefore important to compare data and theory for the prompt and $\chi_{c}$ components separately. This can be done reconstructing the $\chi_{c} \rightarrow J / \psi \gamma$ decay and measuring the fraction of $J / \psi$ that originates from this source.

The $\chi_{c}$ analysis is based on $32,642 \pm 181 J / \psi$ candidates reconstructed in about $20 \mathrm{pb}^{-1}$ of data. To reconstruct the $\chi_{c} \rightarrow J / \psi \gamma$ we combine the $J / \psi$ with photon candidates found in the event. A photon candidate is defined by a central electromagnetic calorimeter tower with energy $E^{\gamma}>1.0 \mathrm{GeV}$ associated with a strip camber cluster and no tracks pointing to the tower. The cluster position and the interaction vertex define the photon direction, this together with the calorimeter energy measurement determines the photon momentum. The mass difference, $\Delta M$, between the dimuon-photon mass and the dimuon mass is shown in Figure 3 , a peak 
of $1230 \pm 71$ events is observed at the mass of about $400 \mathrm{MeV} / \mathrm{c}^{2}$ corresponding to the $\chi_{c}$ states. The width of the signal is about $60 \mathrm{MeV} / \mathrm{c}^{2}$, too large to distinguish the $\chi_{c}^{1}$ and $\chi_{c}^{2}$ separated by $45.7 \mathrm{MeV} / \mathrm{c}^{2}$. The statistics allow to observe a significative signal dividing the sample in four $J / \psi p_{T}$ bins.

We obtain the shape of the background with a Monte Carlo method that uses the real $J / \psi$ events as input. Each charged track in the event is assumed to be a $\pi^{0}, \eta$ or $K_{s}$ in ratios predicted by the measured $K / \pi$ and $\eta / \pi^{0}$ ratios and isospin symmetry. The particles are decayed and the resulting photons simulated through the detector. Applying the $\chi_{c}$ reconstruction to this event gives a $\Delta M$ distribution that is our model for the shape of the background. The number of signal events is extracted fitting the data to the sum of a gaussian and this background shape. To test this model we compared the $\Delta M$ distribution obtained in this way with the one directly obtained from the data, both for dimuon pairs in the sidebands of the $J / \psi$ peak where there is no $\chi_{c}$ signal. The agreement is very good for every $J / \psi p_{T}$ bin.

The photon reconstruction efficiency is obtained using conversion electrons found in the data and correcting for the known differences in detector response between photons and electrons. After correcting for the the photon acceptance and reconstruction efficiency we obtain a fraction of $J / \psi$ from $\chi_{c}$ of $28 \pm 1.6 \pm 6.8 \%$. This fraction includes a contribution from $B \rightarrow J / \psi X$ and $B \rightarrow \chi_{c} X$ that must be removed. We do this using the cross section of $J / \psi$ from $b$ 's measured by CDF and the branching ratios of $B$ mesons to charmonium states measured by CLEO. This correction is small, the fraction of $J / \psi$ from $\chi_{c}$ without the contribution from $b$ 's is $32 \pm 2.0 \pm 8.5 \%$ and is shown as function of $J / \psi p_{T}$ in Figure 4. Figure 5 shows the cross section for $J / \psi$ from $\chi_{c}$ and for direct $J / \psi$, both with the $b$ contribution removed. The $J / \psi$ from $\chi_{c}$ cross section is in agreement with the theoretical calculation while the direct $J / \psi$ data is again a factor $\sim 50$ over the prediction.

We conclude that the color singlet perturbative QCD model of charmonium production fails to describe direct production, both for the $\psi(2 S)$ and the $J / \psi$ by about the same large amount. Contrary to its prediction direct production is the dominant source of prompt $\psi(2 S)$ and $J / \psi$. This data does not exclude the possibility of a contribution from $c \bar{c}$ states above the open charm threshold, but makes this an unlikely explanation for the large excess of direct $\psi(2 S)$ and $J / \psi$. It has been shown that the inclusion of the color octet mechanism establishes agreement with the data, although introducing parameters not calculable within the model and derived from the fit to the data [10].

As noted earlier this analysis has insufficient resolution to distinguish the two $\chi_{c}$ states. A complementary analysis can be done requiring that the photon has converted into an $e^{+} e^{-}$pair. With this method the photon reconstruction uses only tracking information greatly improving the $\chi_{c}$ mass resolution. The efficiency of this reconstruction is low, therefore this analysis utilizes a larger data sample corresponding to $75 \mathrm{pb}^{-1}$. Events are selected requiring the photon to have $p_{T}>1.0 \mathrm{GeV} / \mathrm{c}$ and a conversion vertex separated from the primary interaction vertex by more than $1.0 \mathrm{~cm}$ in the transverse plane. To select prompt $\chi_{c}$ candidates we use only $J / \psi$ reconstructed 
in the SVX and require $c \tau_{\text {pseudo }}<100 \mu m$. The resulting $J / \psi \gamma$ mass distribution is shown in Figure 6 . The $\chi_{c}^{1}$ and $\chi_{c}^{2}$ peaks, with $46 \pm 7$ and $23 \pm 6$ events respectively, are nicely separated. These rates, with a small acceptance correction and the known decay branching ratios, are used to obtain the relative production cross section of:

$$
\frac{\sigma\left(\chi_{c}^{2}\right)}{\sigma\left(\chi_{c}^{1}\right)+\sigma\left(\chi_{c}^{2}\right)}=0.47 \pm 0.08(\text { stat }) \pm 0.02(\text { sys })
$$

\section{$2.3 \Upsilon$}

In the $b \bar{b}$ system CDF has measured the differential cross section for the $\Upsilon$ 's. In $17 \mathrm{pb}^{-1}$ of data we have reconstructed $\sim 1200 \Upsilon(1 S), \sim 300 \Upsilon(2 S)$ and $\sim 200 \Upsilon(3 S)$. The production cross sections measured in the rapidity range $|y|<0.4$ are:

$$
\begin{aligned}
& \sigma(\Upsilon(1 S))=(23.48 \pm 0.99 \pm 2.80) \mathrm{nb}, p_{t}>0.5 \mathrm{GeV} / \mathrm{c} \\
& \sigma(\Upsilon(2 S))=(10.07 \pm 1.01 \pm 1.99) \mathrm{nb}, p_{t}>1.0 \mathrm{GeV} / \mathrm{c} \\
& \sigma(\Upsilon(3 S))=(4.79 \pm 0.64 \pm 0.72) \mathrm{nb}, p_{t}>1.0 \mathrm{GeV} / \mathrm{c}
\end{aligned}
$$

The differential cross sections are shown in Figure 7. The theoretical curve is a leading order QCD calculation that includes the contributions from all the known $\chi_{b}$ states and feed-down from the $S$ states. The measured cross sections are higher than the calculations by a factor $\sim 3$ for the $1 S$ and $2 S$ and $\sim 10$ for the $3 S$. For the $\Upsilon$ 's the experimental analysis, at this time, is not as complete as it is in the charmonium system because the contribution from the $\chi_{b}$ 's has not been separated. The lower statistics have not allowed the reconstruction of the radiative decay of the $\chi_{b}$ yet. Therefore a conclusion on the origin of the disagreement can not be definitively drawn. For the $\Upsilon$ 's the shape of the differential cross section is also not reproduced by the calculation in the low $p_{T}$ region, but it has been shown that including a parton $k_{T}$ smearing agreement in the shape can be obtained [10]. Unfortunately also this effect cannot be quantitatively calculated and must be derived from a fit to the data.

\section{Charmonium Production at Fixed Target}

\section{1 $\quad \mathbf{E 7 8 9}$}

In this experiment the primary $800 \mathrm{GeV} / \mathrm{c}$ proton beam was incident upon a thin, $3 \mathrm{~mm}$ long, gold target. Charged particles resulting from the interaction were magnetically deflected around a beam dump suspended within the first analyzing magnet. A second magnet downstream provided the momentum measurement and confirmed 
the association of the track with the interaction point. Muon detectors were located behind a thick absorber wall [11]. The acceptance was limited to the small Feynman- $x$ range: $-0.03<x_{F}<0.15$.

In the data analysis a pair of opposite sign muons was required and the two tracks were constrained to originate from the target. The resulting mass resolution for the $J / \psi$ was $16 \mathrm{MeV} / \mathrm{c}^{2}$. The differential cross sections were derived from $112649 \pm$ $486 \mathrm{~J} / \psi$ and $1824 \pm 114 \psi(2 S)$. These are shown in Figure 8 compared with the leading order perturbative QCD calculation. While the prediction and the data show reasonable agreement in shape if the proper $k_{T}$ smearing is used, the theoretical calculations have been increased by a $K$ factor equal to 7 for the $J / \psi$ and 25 for the $\psi(2 S)$ in order to get agreement in magnitude [12]. It is interesting to notice that the $K$ factors needed to bring the theoretical calculation in agreement with the CDF data, $p \bar{p}$ at $\sqrt{s}=1.8 \mathrm{TeV}$, are the same of these, $p$-Nucleon at $\sqrt{s}=39 \mathrm{GeV}$, within a factor of two.

\subsection{E705}

This experiment used $300 \mathrm{GeV} / \mathrm{c}$ positive $\left(45 \% \pi^{ \pm}\right.$and $\left.55 \% p\right)$ and negative $\left(98 \% \pi^{ \pm}\right.$ and $2 \% \bar{p}$ ) beams. The beam was aimed at a $33 \mathrm{~cm}$ long lithium target. Two beamline Cerenkov counters tagged the beam particle type on an event by event basis. The large acceptance spectrometer included magnetic analysis of charged tracks, an electromagnetic calorimeter for the photon identification and muon detectors. The Feynman- $x$ acceptance was : $-0.10<x_{F}<0.45$. Reconstruction of the dimuon data yielded a sample of approximately $23000 \mathrm{~J} / \psi$ and $500 \psi(2 S)$ [13].

The $J / \psi$ data sample was used to reconstruct the radiative decays of the $\chi_{c}$ 's. For this analysis the muon pair was combined with all photon candidates, other than those from reconstructed $\pi^{0}$ decays, with $E^{\gamma}>2.5 \mathrm{GeV}$. Figure 9 shows the resulting $\Delta M$ distribution for $\pi^{ \pm}$and proton beams. To measure the relative $\chi_{c}^{1} / \chi_{c}^{2}$ production rate a simultaneous likelihood fit to the two mass plot was performed. The $\chi_{c}^{1}$ and $\chi_{c}^{2}$ resolution function used was derived from the Monte Carlo simulation. Correcting for the known decay branching ratio the result is $\sigma\left(\chi_{c}^{1}\right) / \sigma\left(\chi_{c}^{2}\right)=0.52_{-0.27}^{+0.57}$ for the $\pi^{ \pm}$ beam and $\sigma\left(\chi_{c}^{1}\right) / \sigma\left(\chi_{c}^{2}\right)=0.08_{-0.15}^{+0.25}$ for the proton beam [14].

The suppression of $\chi_{c}^{1}$ production in $p$-Nucleon production, at the low $p_{T}$ typical of fixed target experiments, is predicted by the QCD color singlet model. This is because, at leading order, only gluon-gluon fusion contributes to $\chi_{c}$ production and the $\chi_{c}^{1}$, a $1^{++}$state, can not couple to two gluons. In this case the experimental result is in agreement with the theoretical prediction, on the other hand the two $\chi_{c}$ states in this analysis are not cleanly separated and the uncertainties are large.

This experiment has also performed a thorough study of the sources of $J / \psi$ mesons for each beam particle type. From the number of reconstructed $J / \psi$ and $\chi_{c}$, correcting for the photon acceptance, E705 measures the fraction of $J / \psi$ arising from $\chi_{c}$ decays. Another contribution to $J / \psi$ production is $\psi(2 S)$ production followed by 
decay into final states containing $J / \psi$. To obtain this fraction the measured $J / \psi$ and $\psi(2 S)$ cross sections together with the known decay branching ratios are used. The results are summarized in Table 1 together with the $\psi(2 S) / J / \psi$ cross section ratio. The direct $J / \psi$ is the fraction not from $\chi_{c}$ or $\psi(2 S)$ [15].

\begin{tabular}{|c|c|c|c|c|}
\hline beam & $\sigma\left(\psi^{\prime}\right) / \sigma(\psi)$ & $F_{\chi}^{\psi}$ & direct $\psi$ & from $\psi^{\prime}$ \\
\hline$\pi^{+}$ & $0.12 \pm 0.04$ & $0.40 \pm 0.04$ & $0.54 \pm 0.05$ & $0.064 \pm 0.022$ \\
\hline$\pi^{-}$ & $0.14 \pm 0.03$ & $0.37 \pm 0.03$ & $0.56 \pm 0.03$ & $0.075 \pm 0.017$ \\
\hline$p$ & $0.14 \pm 0.03$ & $0.30 \pm 0.04$ & $0.62 \pm 0.04$ & $0.075 \pm 0.017$ \\
\hline $\bar{p}$ & $0.25 \pm 0.22$ & & & \\
\hline
\end{tabular}

Table 1.

The fraction of $J / \psi$ from $\chi_{c}$ is comparable with what found by CDF at a much higher $\sqrt{s}, F_{\chi}^{\psi}(C D F)=32 \pm 2.0 \pm 8.5 \%$. A large direct $J / \psi$ component is necessary to describe the data, as for CDF and the ratio of $\psi(2 S)$ to $J / \psi$ production is also similar to the CDF value of $0.19 \pm 0.05$.

\section{$3.3 \quad \mathrm{E} 672 / 706$}

This experiment was performed with a $515 \mathrm{GeV} / \mathrm{c} \pi^{-}$beam incident on berillium and copper targets. The spectrometer [16] had the capability of studying high mass dimuon pairs in the Feynman- $x$ range of: $0.10<x_{F}<0.80$. A sample of $9600 \pm 105$ $J / \psi$ and $270 \pm 35 \psi(2 S)$ was obtained.

The production of $J / \psi$ from $\psi(2 S)$ and $\chi_{c}$ was studied. From a sample of $220 \pm$ 43 reconstructed $\psi(2 S) \rightarrow J / \psi \pi^{+} \pi^{-}$decays, correcting for the $\psi(2 S)$ reconstruction efficiency and the known ratio of branching ratio $B r(\psi(2 S) \rightarrow J / \psi X) / B r(\psi(2 S) \rightarrow$ $\left.J / \psi \pi^{+} \pi^{-}\right)$, the fraction of $J / \psi$ from $\psi(2 S)$ was obtained. To study the production from $\chi_{c}$ and reach the mass resolution necessary to separate the $\chi_{c}^{1}$ and $\chi_{c}^{2}$ states only photons that had converted into an $e^{+} e^{-}$pair were used. Figure 10 shows the $\mu^{+} \mu^{-} \gamma$ mass distribution. Two nicely separated peaks corresponding to the $\chi_{c}^{1}$ and $\chi_{c}^{2}$ with $47 \pm 12$ and $37 \pm 11$ events respectively are observed. From this the relative production rate of $\sigma\left(\chi_{c}^{1}\right) / \sigma\left(\chi_{c}^{2}\right)=0.63 \pm 0.25$ was derived together with the fraction of $J / \psi$ from $\chi_{c}^{1}$ and $\chi_{c}^{2}$. Unfortunately this experiment has not collected data with a proton beam that can be used to measure the $\chi_{c}^{1} / \chi_{c}^{2}$ ratio with this precision. The fractions are summarized in Table 2.

\begin{tabular}{|c|c|c|c|c|}
\hline beam & $F_{\chi^{1}}^{\psi}$ & $F_{\chi^{2}}^{\psi}$ & direct $\psi$ & from $\psi^{\prime}$ \\
\hline$\pi^{-}$ & $0.26 \pm 0.07$ & $0.20 \pm 0.06$ & $0.45 \pm 0.09$ & $0.08 \pm 0.02$ \\
\hline
\end{tabular}

Table 2. 
The rate of $\sigma\left(\chi_{c}^{1}\right) / \sigma\left(\chi_{c}^{2}\right)=0.63 \pm 0.25$ corresponds to $\sigma\left(\chi_{c}^{2}\right) /\left(\sigma\left(\chi_{c}^{1}\right)+\sigma\left(\chi_{c}^{2}\right)\right)=$ $0.61 \pm 0.10$, higher, but not too different from the CDF measurement.

This experiment has compared these results with a theoretical calculation [17] and good agreement was found. The model used in this calculation includes effect not included in the standard Perturbative QCD one.

\section{Conclusions}

Recent data on $J / \psi, \psi(2 S), \chi_{c}$ and $\Upsilon$ production has unraveled surprises. The theoretical model based on perturbative QCD, previously believed to give a reasonable description of quarkonia production, dramatically fails to reproduce the data. By disentangling all production sources (from $b$ 's and direct, for the $\psi(2 S)$; from $b$ 's from $\chi_{c}$ 's and direct, for the $\left.J / \psi\right)$ CDF found that direct production is the main production mechanism of prompt $J / \psi$ and $\psi(2 S)$ in disagreement with the expectations by a factor of 50. Fixed target data on charmonium production show a remarkably similar patter and have been available for a long time before the discovery of the "CDF $\psi(2 S)$ anomaly", but, for reasons hard to understand within a Galileian approach, these data did not stimulate much theoretical interest.

From the experimental point of view more can be learned at collider experiments by studying production in the $b \bar{b}$ system separating direct $\Upsilon$ from feed-down from $\chi_{b}$. Fixed target experiments have the possibility of testing a crucial prediction of the theory, namely the suppression of the $\chi_{c}^{1}$ in proton-Nucleon production. At the moment this prediction seem to be confirmed by the experiments but the data are not yet clean enough for a firm conclusion. 


\section{References}

[1] R. Baier and R. Ruckl, Z.Phys. C19 (1983) 251

E.W.N. Glover, A.D. Martin, W.J. Stirling, Z.Phys. C38 (1988) 473.

[2] E.Braaten and T.C. Yuan, Phys.Rev.Lett. 71 (1993) 1673.

[3] E. Braaten, et al., Phys.Lett. B333 (1994) 548.

M.Cacciari, M.Greco Phys. Rev. Lett. 73 (1994) 1586.

[4] UA1 Coll.,C. Albajar et al., Phys.Lett. B256 (1991) 112.

CDF Coll.,F. Abe et al., Phys.Rev.Lett. 69 (1992) 3704.

CDF Coll.,F. Abe et al., Phys.Rev.Lett. 71 (1993) 2537.

[5] C. Korkoumelis et al., Phys.Lett. B81 (1979) 405.

Y. Lemoigne et al., Phys.Lett. B113 (1982) 509.

S.R. Hahn et al., Phys. Rev. D30 (1984) 671.

D.A. Bauer et al., Phys.Rev.Lett. 54 (1985) 753.

[6] F. Abe et al., Nucl. Instrum. Methods A271 (1988) 387

[7] D. Amedei et al., Nucl. Instrum. Methods A350 (1994) 73

[8] F.E.Close, Phys.Lett. B342 (1995) 369.

D.P.Roy and Sridhar, Phys.Lett. B345 (1995) 537.

P.Cho and M.Wise, Phys.Lett. B346 (1995) 129.

[9] E.Braaten and S.Fleming Phys.Rev.Lett. 74 (1995) 3327.

[10] M.Mangano, these proceedings.

M.Cacciari et al. CERN-TH/95-129.

[11] D.E.Jaffe et al., Phys. Rev. D40 (1989) 2777.

[12] M.H.Schub et al. FERMILAB-PUB-95/058-E, Submitted to Phys. Rev. D

[13] L.Antoniazzi et al., Phys. Rev. D46 (1992) 4828.

[14] L.Antoniazzi et al., Phys. Rev. D49 (1994) 543.

[15] L.Antoniazzi et al., Phys. Rev. Lett. 70 (1993) 383.

[16] G.Alverson et al., Phys. Rev. D480 (1993) 5.

R.Jesik et al., Phys. Rev. Lett. 74 (1995) 495.

[17] G.A.Schuler CERN-TH.7170/94 submitted to Physics Reports. 


\section{List of Figures}

$1 c \tau_{p s e u d o}$ distribution for the $\psi(2 S)$ decomposed into prompt (unshaded), from $b$ decays (hatched), and background (dark shading). . . . . . 12

$2 \quad \psi(2 S)$ differential cross section. The curves are NLO QCD calculations. 13

3 Mass difference $M\left(\mu^{+} \mu^{-} \gamma\right)-M\left(\mu^{+} \mu^{-}\right)$for the $\psi$ signal region in $40 \mathrm{MeV} / \mathrm{c}^{2}$ bins. The points show the data histogram, the shaded histogram is the background shape predicted by our background Monte Carlo. The solid line is the fit to the data of a gaussian signal plus the background histogram. . . . . . . . . . . . . 14

4 Fraction of $J / \psi$ from $\chi_{c}$ as function of the $J / \psi p_{T}$ with the contribution

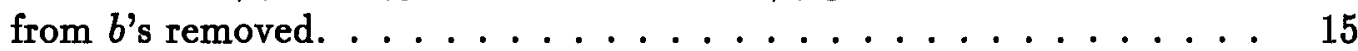

$5 \quad J / \psi$ differential cross sections as a function of $J / \psi p_{T}$ with the contribution from $b$ 's removed; the circles show the direct component, the squares correspond to $J / \psi$ from $\chi_{c}$ and the triangle is the sum. The curves are the NLO QCD calculations. . . . . . . . . . . . 16

6 The $J / \psi \gamma$ mass distribution, based on tracking measurement via photon conversion, for prompt $J / \psi \ldots \ldots \ldots \ldots \ldots \ldots$

$7 \Upsilon$ differential cross sections as a function of $p_{T}$ The curves are the LO QCD calculations. . . . . . . . . . . . . . . 18

8 Differential cross section for $J / \psi$ and $\psi(2 S)$ measured by the E789 experiment. The leading-order predictions with $K$ factors of 7 and 25 for the $J / \psi$ and $\psi(2 S)$ respectively are also shown. . . . . . . .

9 The $M\left(\mu^{+} \mu^{-} \gamma\right)-M\left(\mu^{+} \mu^{-}\right)$distribution for $\pi^{ \pm}$and $p$ induced reactions obtained by the E705 experiment. The smooth curves are from the likelihood fit, and the insets show the background subtracted $\chi_{c}$ signal. 20

10 The $J / \psi \gamma$ mass distribution, based on tracking measurement via photon conversion, obtained by the E672/706 experiment. The solid line shows the fit to the data. The lower histogram shows the background subtracted signal. . . . . . . . . . . . . 


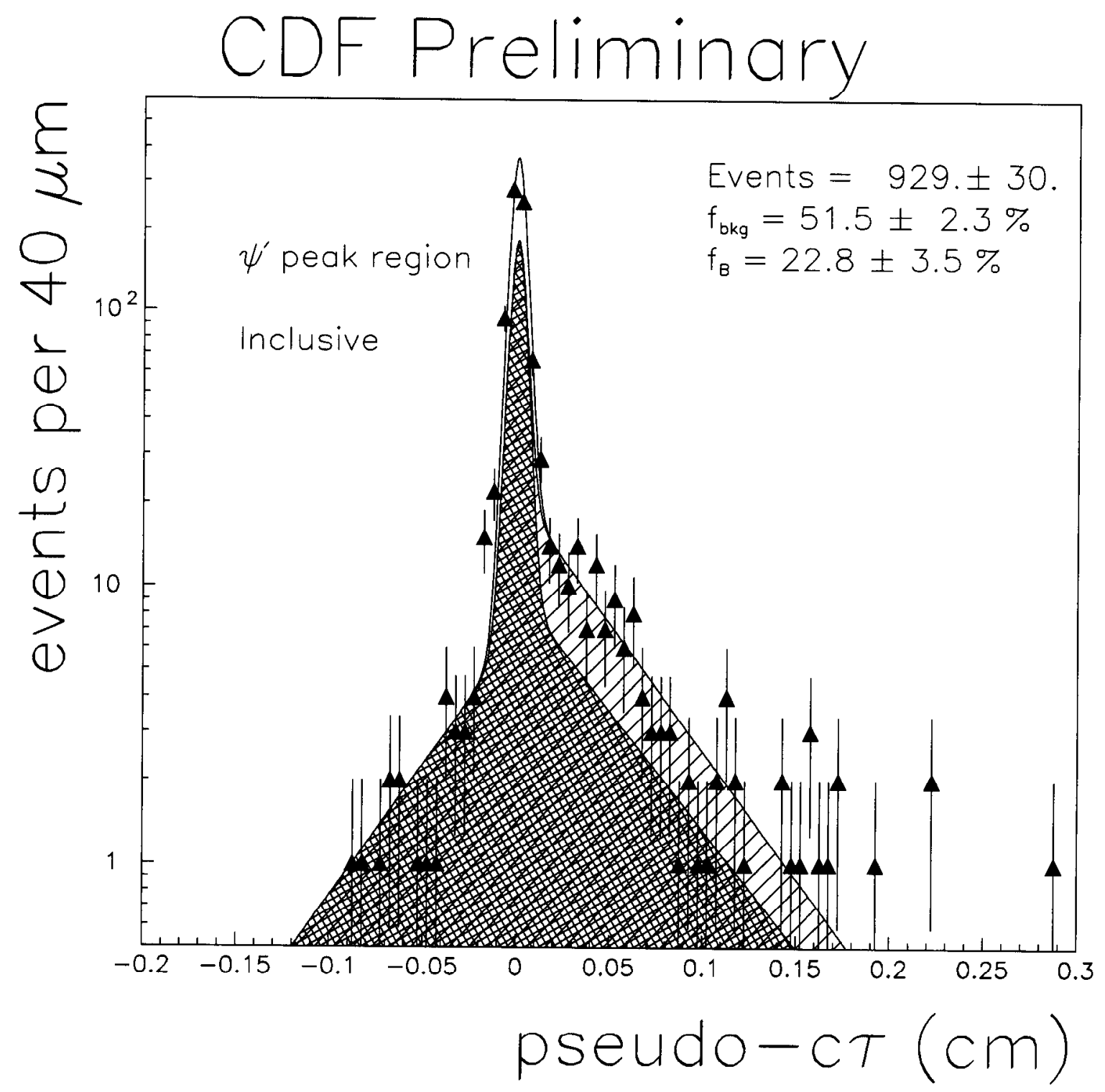

Figure 1: $c \tau_{\text {pseudo }}$ distribution for the $\psi(2 S)$ decomposed into prompt (unshaded), from $b$ decays (hatched), and background (dark shading). 
CDF Preliminary

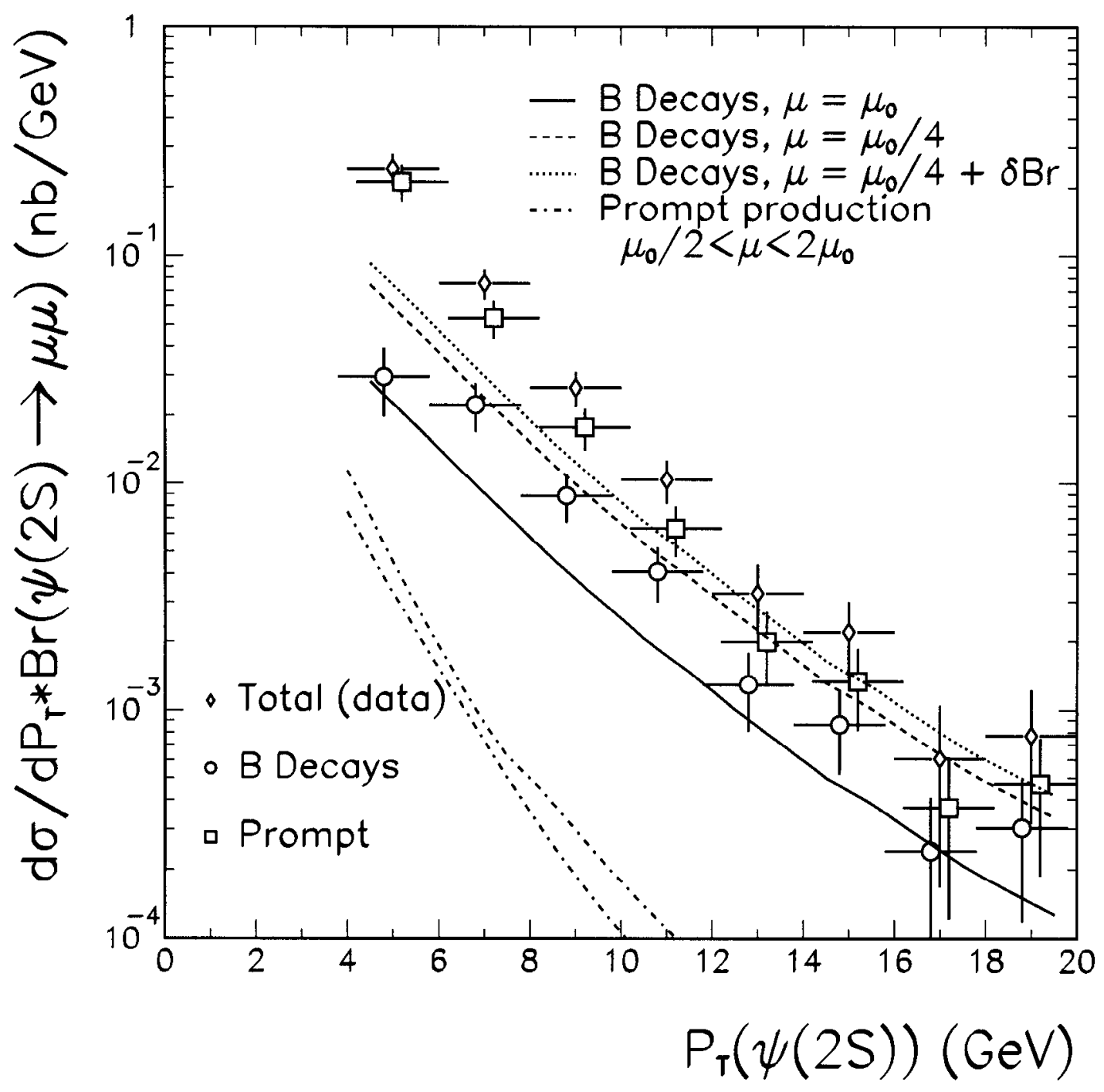

Figure 2: $\psi(2 S)$ differential cross section. The curves are NLO QCD calculations. 


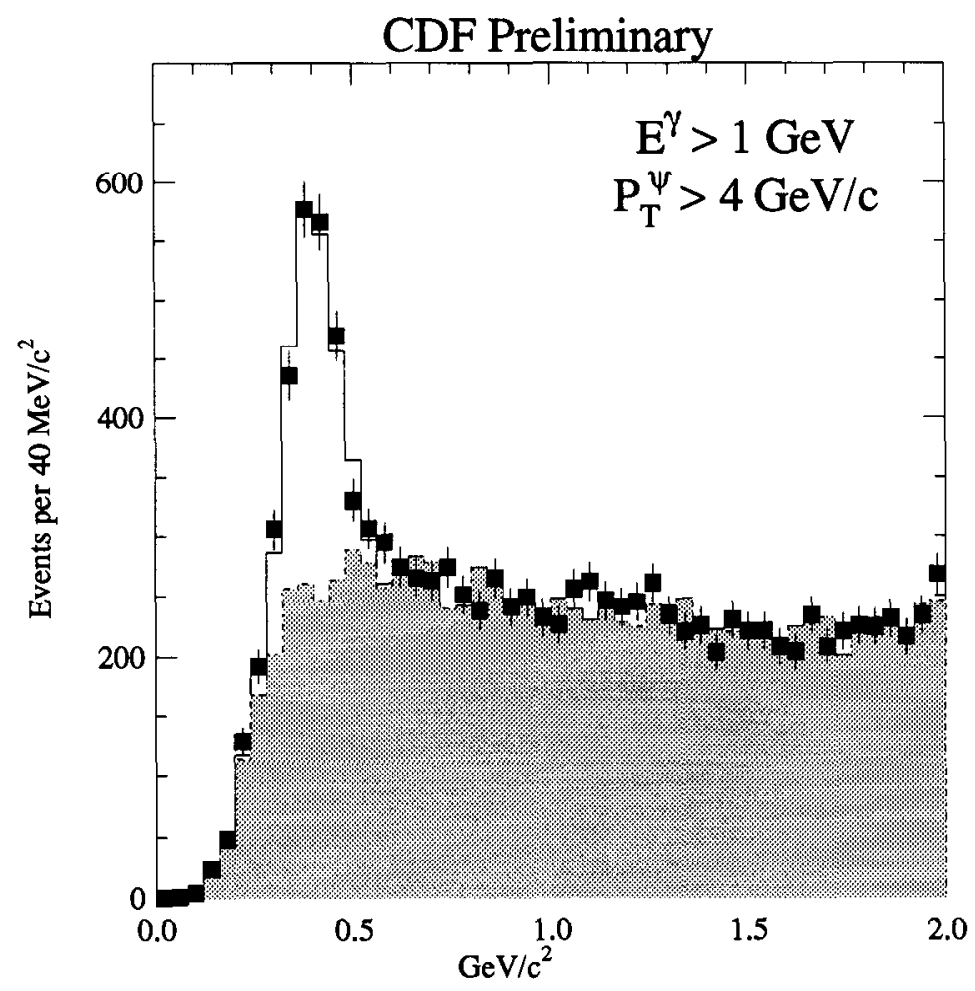

Figure 3: Mass difference $M\left(\mu^{+} \mu^{-} \gamma\right)-M\left(\mu^{+} \mu^{-}\right)$for the $\psi$ signal region in $40 \mathrm{MeV} / c^{2}$ bins. The points show the data histogram, the shaded histogram is the background shape predicted by our background Monte Carlo. The solid line is the fit to the data of a gaussian signal plus the background histogram. 
CDF Preliminary

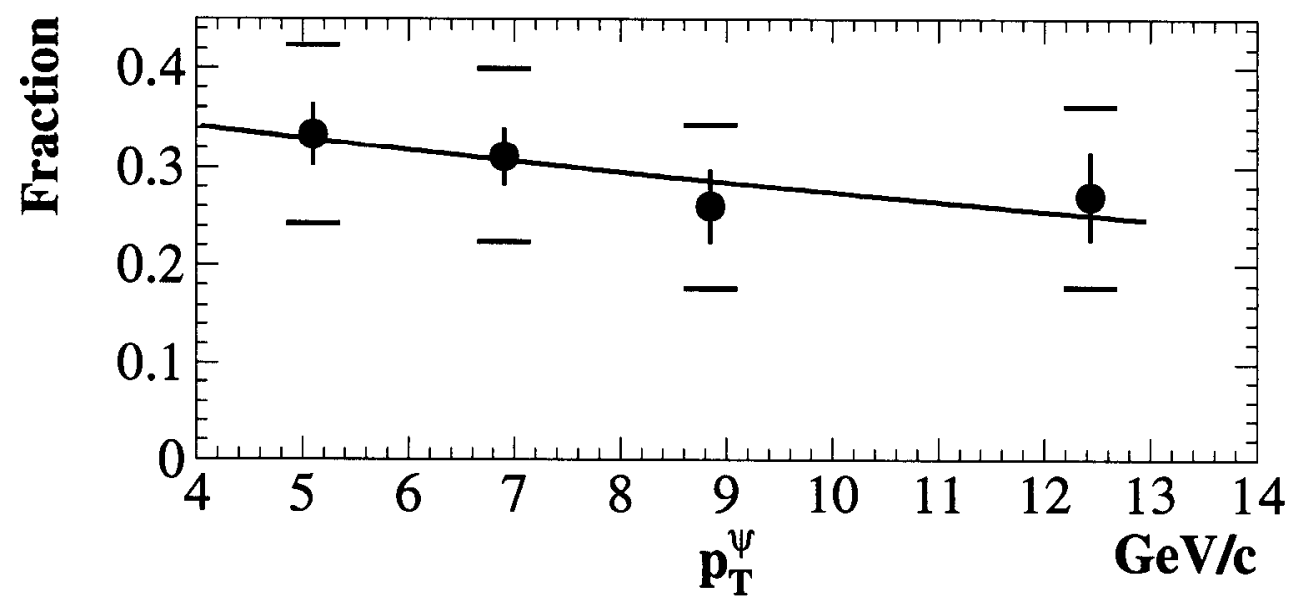

Fraction of $J / \psi$ from $\chi_{c}$ vs $p_{T}^{J / \psi}$ with the contribution from b's removed.

\begin{tabular}{|c|c|}
\hline$P_{T}^{\psi}>4.0 \mathrm{GeV} / \mathrm{c}$ & $0.323 \pm 0.020 \pm 0.085$ \\
\hline $4.0<P_{T}^{\psi}<6.0 \mathrm{GeV} / \mathrm{c}$ & $0.333 \pm 0.031 \pm 0.085$ \\
\hline $6.0<P_{T}^{\psi}<8.0 \mathrm{GeV} / \mathrm{c}$ & $0.311 \pm 0.029 \pm 0.083$ \\
\hline $8.0<P_{T}^{\psi}<10.0 \mathrm{GeV} / \mathrm{c}$ & $0.260 \pm 0.038 \pm 0.074$ \\
\hline$P_{T}^{\psi}>10.0 \mathrm{GeV} / \mathrm{c}$ & $0.270 \pm 0.045 \pm 0.080$ \\
\hline \hline
\end{tabular}

Figure 4: Fraction of $J / \psi$ from $\chi_{c}$ as function of the $J / \psi p_{T}$ with the contribution from $b$ 's removed. 


\section{CDF PRELIMINARY}

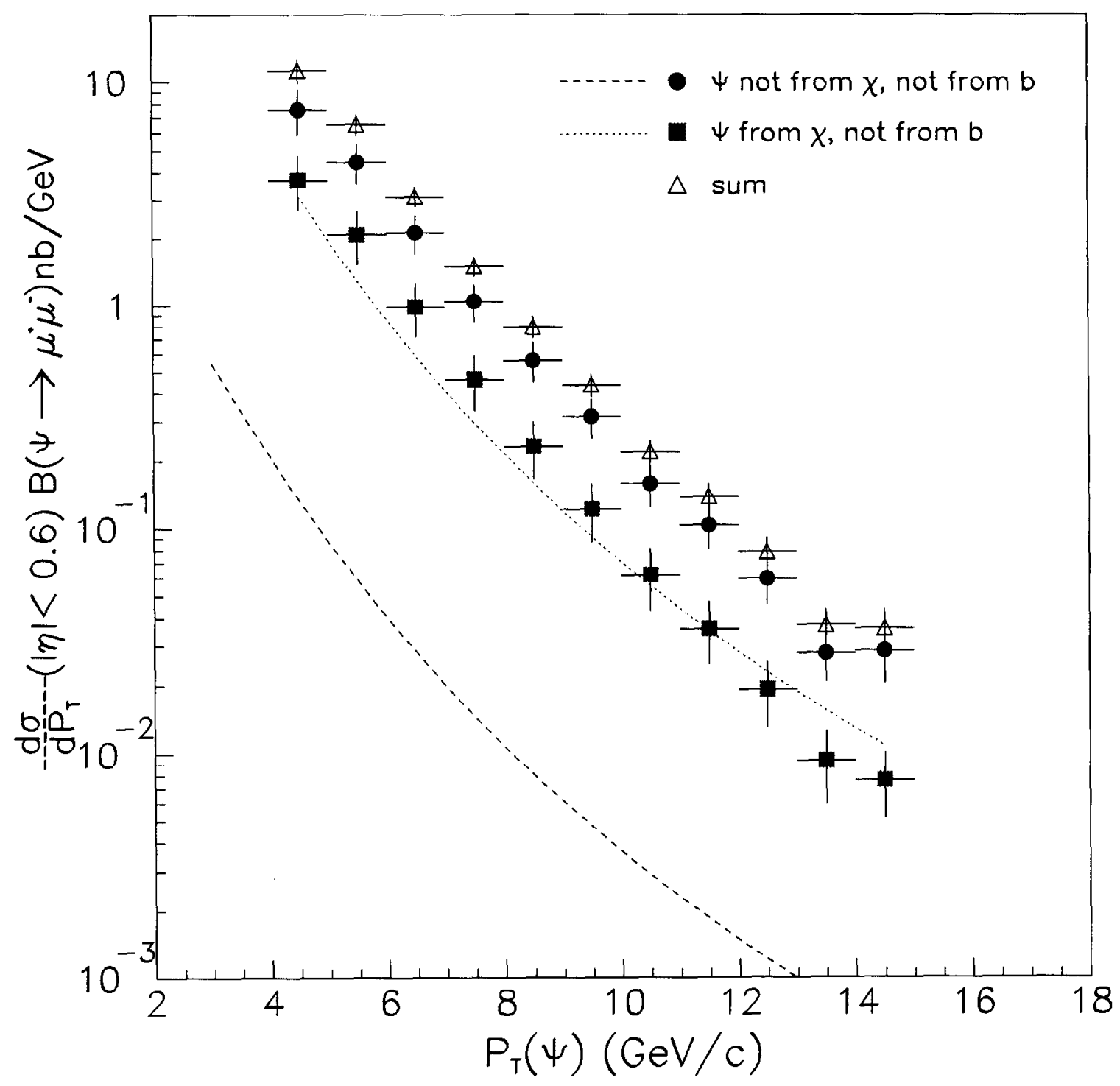

Figure 5: $J / \psi$ differential cross sections as a function of $J / \psi p_{T}$ with the contribution from $b$ 's removed; the circles show the direct component, the squares correspond to $J / \psi$ from $\chi_{c}$ and the triangle is the sum. The curves are the NLO QCD calculations. 


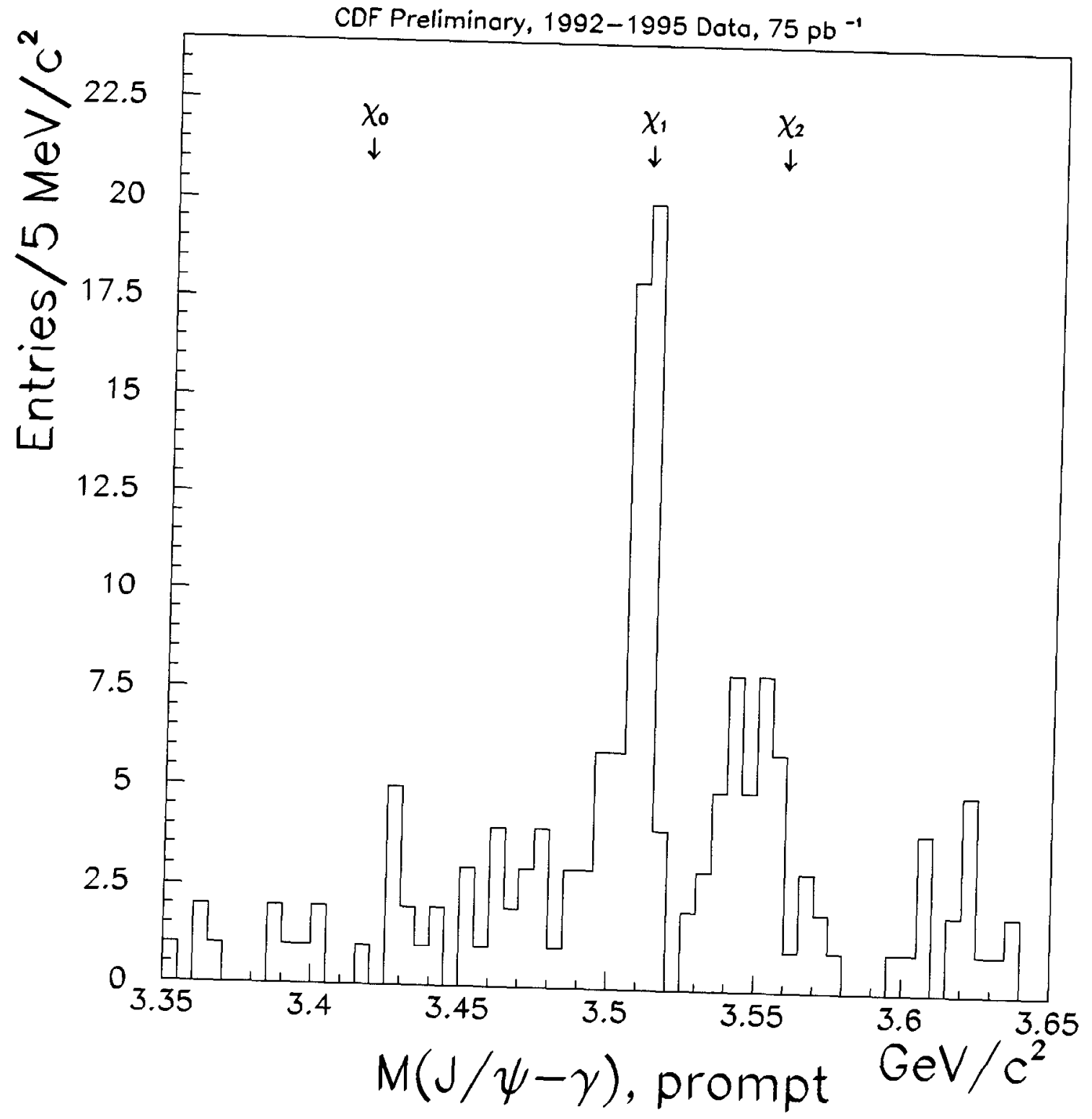
Figure 6: The $J / \psi \gamma$ mass distribution, based on tracking measurement via photon
conversion, for prompt $J / \psi$. 

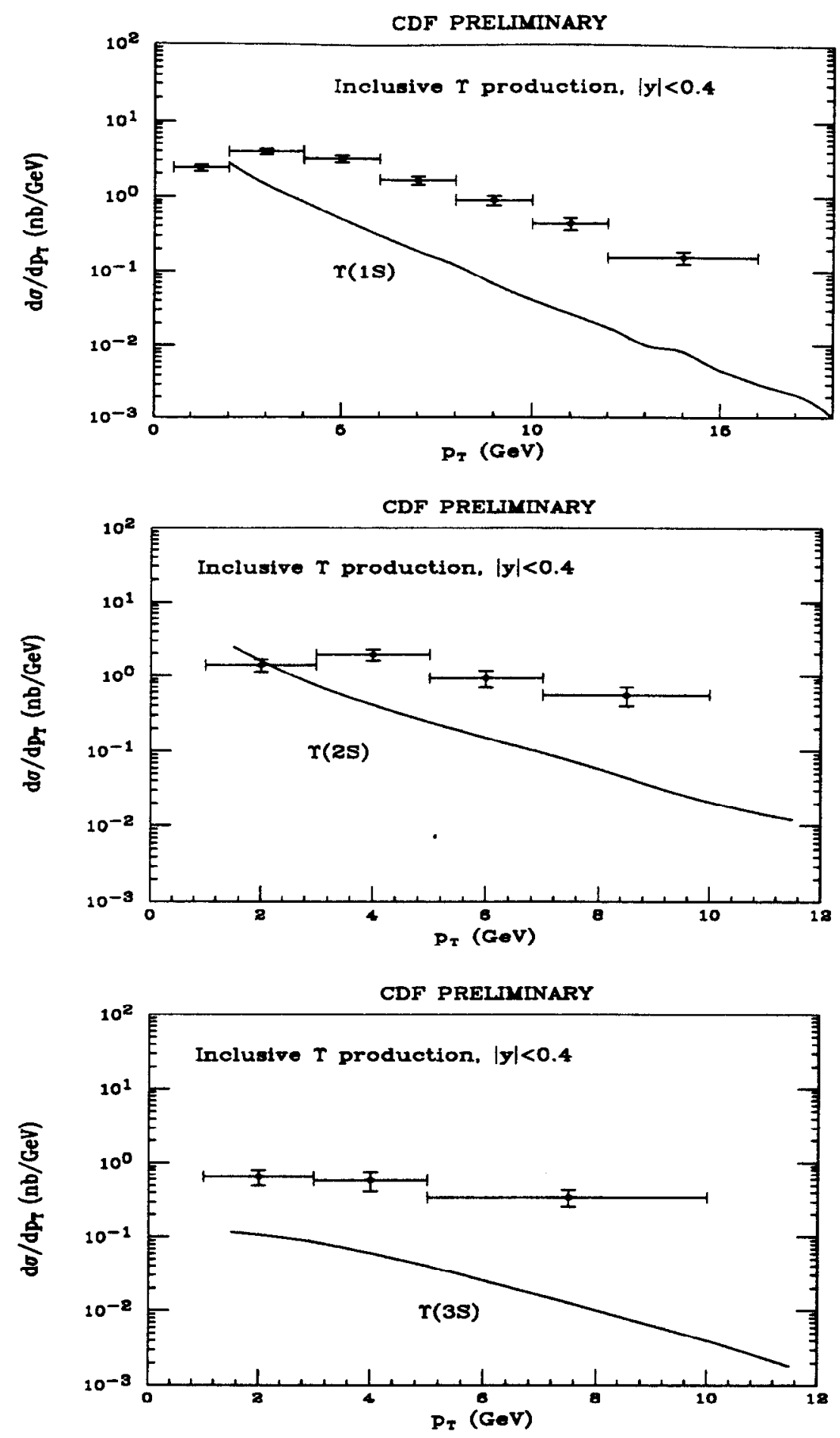

Figure 7: $\Upsilon$ differential cross sections as a function of $p_{T}$ The curves are the LO QCD calculations. 


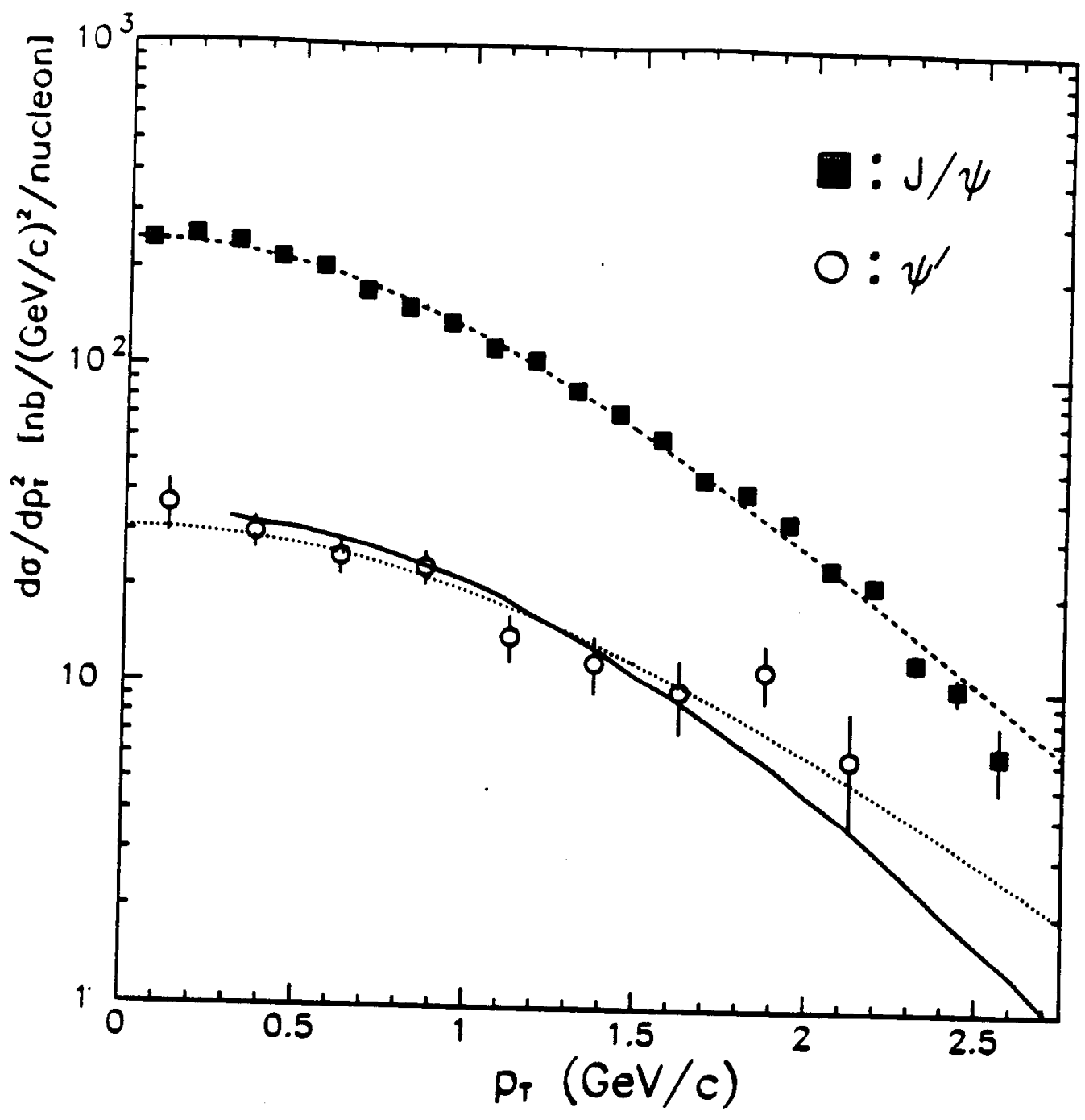

Figure 8: Differential cross section for $J / \psi$ and $\psi(2 S)$ measured by the E789 experiment. The leading-order predictions with $K$ factors of 7 and 25 for the $J / \psi$ and $\psi(2 S)$ respectively are also shown. 


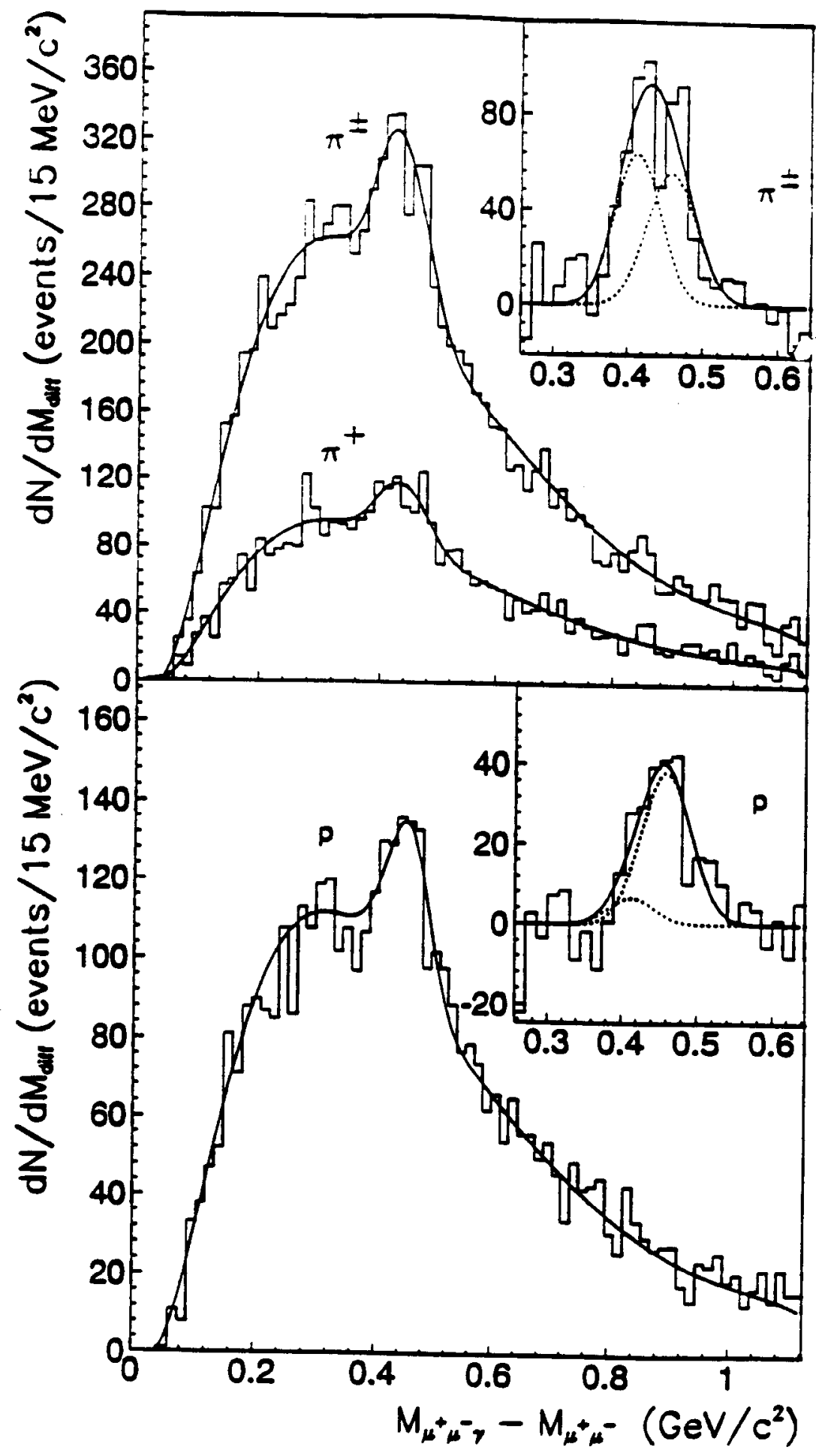

Figure 9: The $M\left(\mu^{+} \mu^{-} \gamma\right)-M\left(\mu^{+} \mu^{-}\right)$distribution for $\pi^{ \pm}$and $p$ induced reactions obtained by the E705 experiment. The smooth curves are from the likelihood fit, and the insets show the background subtracted $\chi_{c}$ signal. 


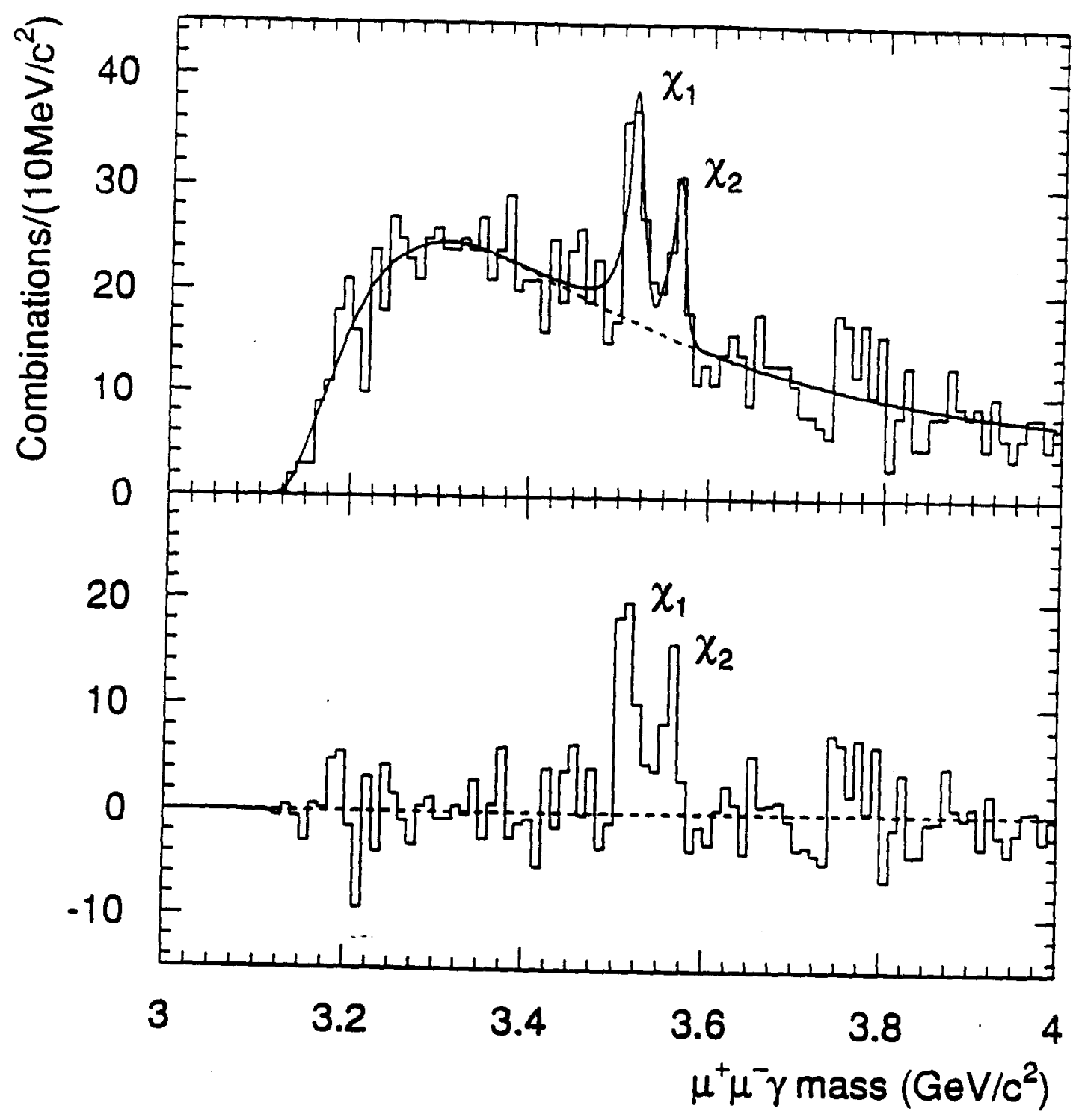

Figure 10: The $J / \psi \gamma$ mass distribution, based on tracking measurement via photon conversion, obtained by the E672/706 experiment. The solid line shows the fit to the data. The lower histogram shows the background subtracted signal. 